\title{
Enseñar derecho de forma colaborativa. Ensayo sobre una primera experiencia
}

\section{Teaching collaboratively law. Essay on a first experience}

\author{
Carmen Gloria Pérez Villar ${ }^{1}$
}

\begin{abstract}
Resumen: el estado actual de la enseñanza del derecho muestra la presencia aún fuerte de la clase magistral como metodología recurrente. Frente a este hecho, se plantea la necesidad de innovar en la enseñanza y así desarrollar en los estudiantes otras competencias, además del saber escuchar. La propuesta de la autora es una metodología participativa o colaborativa, que se caracteriza por la participación del estudiante en su proceso de aprendizaje y por desplazar el foco del proceso desde la enseñanza al aprendizaje. Los aspectos que hay que privilegiar en esta metodología son: el perfil de egreso, el desarrollo de competencias, ceñirse a un programa de estudio y seleccionar actividades relevantes, entre otros.
\end{abstract}

Palabras clave: metodología colaborativa, enseñanza del Derecho, metodología jurídica.

Abstract: the current condition of legal education still shows the strong presence of the lecture as a recurring methodology. Given this fact, there is a need for innovation in teaching and developing other skills in students (besides knowing to listen). The aim of the author is a participatory or collaborative methodology, which is characterized by student participation in the learning process and by shifting the focus of the process from teaching to learning. The aspects to be priority in this methodology are the graduate profile, skills development, fitting to a program of study and selection relevant activities, among others.

Key words: collaborative methodology, law teaching, legal methodology.

\section{Presentación}

Los cambios en la enseñanza del Derecho, producto de las nuevas perspectivas educativas basadas en la adquisición de competencias y no sólo de conocimientos, o nuevo rol profesionalizante de la educación universitaria, ha traído algunas dificultades para quienes nos hemos dedicado a la docencia pero que, sin embargo, no nos educamos con ese sistema, ni tenemos formación pedagógica para enfrentar las metodologías activas o colaborativas necesarias para enfrentar este nuevo esquema.

En estas líneas pretendemos exponer nuestra humilde experiencia, en el entendido que podría orientar a quienes se inician en este camino, destacando especialmente los

\footnotetext{
${ }^{1}$ Licenciada en Ciencias Jurídicas, Universidad Católica de Valparaíso, Magíster en Derecho por la Universidad de Valparaíso, profesora de Derecho Internacional Privado en la Universidad de Valparaíso. Correo electrónico: carmengloriaperez@uvach.cl
} 
aspectos positivos que pueden surgir de una experiencia que, en principio, para los novatos en materias pedagógicas, podría ser abrumadora ${ }^{2}$.

Como decía, para quienes no tenemos formación pedagógica -como ocurre con muchos colegas en las aulas universitarias-, desde la nomenclatura hasta la metodología pueden parecer demasiado complejas, por ello, si algún valor puede tener este trabajo es graficar en términos simples y coloquiales los pasos, que, en nuestra opinión, son fundamentales de identificar al enfrentar las metodologías activas o colaborativas.

Concordando con lo que señala un autor creemos que:

En primer lugar, y aunque parezca muy elemental, se debe tener claro qué se pretende lograr con el proceso de enseñanza - aprendizaje, por qué alguien desea someterse a este proceso complejo y, a veces, doloroso para intentar dominar una disciplina y cuál es el compromiso de la entidad educativa en ese proyecto personal. Esto debiera tenerlo claro tanto el estudiante como el profesor que, en último término, es la herramienta para concretar estos anhelos. Sin embargo, paradojalmente, ello no siempre ocurre así, y, en no pocas oportunidades, por distintas razones -unos por ser unos muy jóvenes e inmaduros y otros por ser los otros mayores y prejuiciados- las partes en esta ecuación no logran el adecuado equilibrio.

Una doctrina española parece estar describiendo la situación que muchas veces ocurre en nuestras aulas. Esto es, el fracaso universitario y la pérdida del prestigio social de la educación universitaria, lo que fomenta una especie de espiral entre el desinterés entre los estudiantes por su formación y el correlativo fracaso universitario. Nos dice el autor:

Acusamos, en ocasiones injustamente a los estudiantes de ser mediocres, de que no saben comprender lo que leen, de que no saben redactar e incluso llegamos a reprochar la falta de vocabulario básico. [...] Son muchos los factores de este fracaso, algunos fuera de nuestro alcance: afectivos, socioeconómicos, etc. Otros son puramente académicos: organización académica, profesorado, etc. Algunos de los factores que se citan son: la desvinculación de los contenidos curriculares con el entorno y posibilidades laborales; carencias graves en las estrategias y técnicas de estudio, utilización de bibliotecas, manejo de información e Internet, trabajo en equipo, etc.; escasa utilización de la moderna tecnología y de recursos didácticos en la práctica docente. [...] En lo que toca al docente, el profesor de derecho debe saber impartir tanto una docencia integral, significativa y crítica de los conocimientos, como una docencia formativa de capacidades intelectuales. ${ }^{3}$

Continuando la cita, el autor nos dice: "[...] si la tarea básica del profesor es formar, intentemos al menos despertar su curiosidad y desarrollar sus habilidades. Creemos que el uso de herramientas múltiples en la enseñanza puede transformar el aula: el aprender haciendo, el uso de la tecnología, la aportación de otros profesionales, etc."4

${ }^{2}$ El presente trabajo no habría sido posible sin la capacitación recibida en el curso de especialización "Certificado en educación superior" impartido por Laureate International Universities, Universidad de Las Américas.

${ }^{3}$ REYES, María y LEAL, María. El aprendizaje del derecho en el nuevo espacio europeo de enseñanza superior. Sevilla: Mergablum, 2006. Pp. 53-55.

${ }^{4}$ Ibid. 
Ahora bien, cada vez que una institución tan importante como la universidad, plantea reformas relevantes, como ocurre con el nuevo modelo educativo basado en competencias, donde ya no basta con entregar sólo conocimientos sobre la disciplina y destrezas para adquirir y producir más y mejor conocimiento, sino que también hay que entregar competencias y destrezas para el desempeño profesional. $\mathrm{O}$ sea, habilidades para ser un buen trabajador que satisfaga los índices de empleabilidad que exige el mercado laboral, nos damos cuenta de que la forma de enseñar no puede ser la misma.

Si en el paradigma tradicional de enseñanza podíamos transmitir conocimientos mediante una clase magistral, hoy que debemos enseñar a ser profesionales competentes, ya no parece tan adecuada esa metodología y queda mejor el estilo del "aprender haciendo". Esto que es evidente en carreras eminentemente prácticas como Medicina o Arquitectura, no lo ha sido en carreras como Derecho. Si bien, podemos ver claramente que un médico para ser un buen cirujano requiere más que clases magistrales, lo mismo que un buen arquitecto, quien debe ser capaz de elaborar proyectos ejecutables una vez egresado de la Universidad, en el caso del estudiante de derecho tradicional esto no estaba tan claro. Así parecía que bastaba conocer sólo la norma jurídica, en sus distintas dimensiones, ojalá bien explicada por quien se maneja en ello, fruto del estudio o la experiencia -el gran maestro-, sin considerar los distintos roles que puede desempeñar un abogado, donde no siempre el acopio de información es lo más relevante para un buen desempeño profesional. De hecho, quizás uno de los roles más fundamentales del abogado sea la resolución de conflictos y es aquí donde, justamente, más que conocer la "ley" resaltan otras habilidades o competencias que pueden marcar la diferencia para hacer de un egresado un profesional exitoso.

La enseñanza del Derecho hace tiempo que ha venido preocupando a algunos académicos, así por ejemplo nuestro querido y recordado profesor Gonzalo Figueroa Yánez lo venía planteando desde la década de 1970 y quizás desde antes -a modo de ejemplo citamos un documento suyo sobre "El estado actual de la docencia y de la investigación en Derecho",5-, donde ya se decían estas mismas cosas. También en países como España hacia donde siempre miramos en materia de Derecho- han emprendido, en su momento, reformas contundentes, con distintos resultados, de lo que da cuenta alguna literatura que ha llegado a nuestras manos, como "La reforma de los estudios de Derecho. El nuevo plan de estudio: su valoración y análisis histórico y comparado" " y "El aprendizaje del Derecho en el nuevo espacio europeo de enseñanza superior" ${ }^{\text {"7 }}$. También el tema ha sido abordado por nuestros vecinos, a modo de ejemplo citamos "Los principios y la enseñanza del derecho privado" 8 .

Ahora bien, el desafío que han tomado algunas universidades en Chile, con el apoyo financiero de proyectos específicos, es potente $\mathrm{y}$, aunque nos presente algunas dificultades

\footnotetext{
5 FIGUEROA, Gonzalo. "El estado actual de la docencia y de la investigación en derecho”. En: Documento de trabajo No 283. Santiago: Corporación de Promoción Universitaria, 1983.

${ }^{6}$ BUJAN, Federico. La reforma de los estudios de derecho. El nuevo plan de estudio: su valoración y análisis histórico y comparado. Madrid: Dykinson, 1992.

${ }^{7}$ REYES, María y LEAL, María. Ibíd.

${ }^{8}$ LIMODIO, Gabriel. Los principios y la enseñanza del derecho privado. Buenos Aires: Editorial de la Universidad Católica Argentina, 2008.
} 
a los académicos, debemos tomarlo como una oportunidad para corregir antiguas falencias del sistema tradicional.

Es esa experiencia inicial de cambio la que quiero compartir, para mostrar una opción o posibilidad a quienes son novatos en metodologías activas, y a los cuales podría ayudar a dar los primeros pasos en la implementación de prácticas simples y ejercicios elementales que no signifiquen gran dificultad ni para los estudiantes ni para el docente.

\section{Algunas prevenciones}

A continuación planteamos algunas cuestiones que estimamos fundamentales, respecto de las cuales podemos tener distintos niveles de entendimiento y comprensión y que es bueno sincerar, para ver en qué medida podemos enfrentar los cambios que se nos proponen, de mejor manera:

Una cuestión fácil: entender los paradigmas de la contemporaneidad educativa

- La sociedad global: No hay área de la cultura y del conocimiento que se encuentre al margen de los procesos de mundialización, los que han significado cambios arrolladores en la forma de vida de las personas, paradigma que se ve reflejado en toda la literatura del siglo XXI.

- Las nuevas tecnologías. No podemos desconocer, especialmente en el área de las comunicaciones, que la tecnología ha sido un elemento fundamental y catalizador del proceso de mundialización. La avalancha de información, en todos los ámbitos, difícil de manejar, con necesidad de seleccionar y discriminar, no ha puesto, a los académicos en la necesidad de replantear nuestro rol en el proceso de enseñanza.

- Democratización en la producción y distribución de información. Lo que Internet ha significado en el mundo de las comunicaciones quizás no tenga parangón en la historia, así los documentos más exclusivos, clasificados y secretos de todo tipo (científicos, técnicos, artísticos, políticos, etc.) están disponibles en la red para quien esté "conectado". Además, hay espacio para todo el que quiera aportar nueva o mejor información, con lo que la "transparencia" en todos los ámbitos aumenta sus posibilidades de ser cada vez más real.

- Los estudiantes de hoy. No los conocemos lo suficiente por su gran diversidad. Ello implica distintos niveles etarios, socioeconómicos, conductuales, culturales, etc. Los jóvenes se manejan bien en lo tecnológico, "aprietan bien las teclas" pero tienen dificultades en otras áreas como las matemáticas básicas, el lenguaje y la escritura. Los adultos manejan mejor las "operaciones básicas" pero tienen mayores dificultades en lo tecnológico, cuestión que también afecta a los académicos.

- El cambio de foco en el proceso de enseñanza. La edad, carácter y personalidad de los estudiantes no es un elemento indiferente a la hora de planificar la enseñanza. Si la educación universitaria, gracias a las políticas de mercado, se ha puesto a disposición de 
todos los potenciales interesados, no se puede desconocer luego las especiales características de estos nuevos "clientes". Por lo demás, está claro que no todos los estudiantes o personas aprenden de la misma manera. Por ejemplo, en la clasificación de Kolb $^{9}$ se distingue a aquellos que aprenden: i) mediante la experiencia concreta, que es la participación directa en una situación real o simulada (alumno activo); ii) a través de la reflexión, donde se pone sentido a lo observado (alumno reflexivo); iii) mediante la abstracción o conceptualización (alumno teórico), y iv) mediante la experimentación, donde se pone a prueba la teoría (alumno pragmático).

De hecho existe un enfoque educativo que ha irrumpido con bastante ímpetu en el ambiente educacional, destinado a valorar y a respetar a cada estudiante en su diversidad y a atribuir valor a la diferencia, por ser un reflejo de la sociedad en que vivimos 10.

- Aumento de las desigualdades sociales. Hoy podemos tener en nuestra sala, lo mismo estudiantes privilegiados que han podido acceder a una educación de calidad, que cuentan con soporte tecnológico y de conexión ilimitado, que se manejan en idiomas y posean una gran cultura vivencial, frente a otros, que siendo primera generación universitaria se van a encontrar con dificultades adicionales para integrarse al mundo universitario.

- El rol del docente. Los cambios que hemos enunciado no significan un desplazamiento hacia una posición más desmejorada del docente, por el contrario, enseñar competencias requiere de la especial guía y experiencia del profesor. Transformar, por ejemplo, el mar de datos que nos brindan las nuevas tecnologías de la información en un río navegable es una función indispensable para llegar a buen puerto y requiere de las competencias adecuadas para ello.

- El nuevo profesional. Quizás la competencia más importante y transversal que deba asumir el docente, es precisamente enseñar a los estudiantes a aprender y a aprehender por sí mismos para el presente y para el futuro. Estas competencias que los profesionales de hoy necesitan dominar, deben venir establecidas en el perfil de egreso, que es un dato que debemos tener presente los docentes para poder orientar y guiar todo nuestro actuar.

- La enseñanza colaborativa. Las nuevas necesidades en la formación universitaria -que ahora debe entregar, además de conocimiento, competencias-, son una combinación compleja. Si a ello sumamos que para lograr estos nuevos objetivos debemos adoptar nuevas formas de enseñanza a las que se llama "participativas o colaborativas" tenemos al docente situado en centro de la atención, a pesar que el centro del proceso educativo se ha traslado desde la enseñanza al aprendizaje.

Una cuestión de mediana dificultad: conocer e internalizar las nuevas metodologías de enseñanza

Se trata, igualmente, de una competencia que el docente debe adquirir con preparación, práctica y entrenamiento, la que debe asumirse como complementaria con las

\footnotetext{
${ }^{9}$ Un esquema básico puede verse en: http://maestrosycontextos.blogdiario.com/1156093080/ [consultado en abril 2012]
} 
fórmulas tradicionales que cada académico desarrolla, pues sería ilusorio pretender sustituir de un día para otro toda una fórmula de enseñanza en que ha sustentado nuestra tradición de enseñanza universitaria, especialmente en el área del Derecho. Estos cambios traen aparejado un sustrato teórico que se funda en el traspaso de la atención desde el profesor hacia el estudiante. Así,

el giro progresivo desde la enseñanza al aprendizaje se ha identificado en la literatura pedagógica como un cambio de paradigma, entendido como la transición desde un paradigma centrado en el profesor y en sus métodos de enseñanza a otro más orientado al aprendizaje y a la construcción activa del conocimiento por parte del sujeto que aprende $^{10}$.

Es evidente, según dice el autor recién citado,

que no basta con saber muchas o más cosas, sino que hay que saberlas bien, de manera personalizada e integrada, de tal forma que sea posible su posterior generalización y aplicación a otros contextos. [...] La equiparación reductora del aprendizaje a la mera adquisición de conocimientos ha desatendido aspectos de la persona igualmente importantes y ha centrado la enseñanza en la transmisión de contenidos de carácter preferentemente conceptual $^{11}$.

Por ello, concordamos con el autor citado, en el sentido de que hoy, efectivamente, es necesario ir más allá del saber más y del saber mejor para llegar a ser capaces -los estudiantes-, de enfrentarse cognitiva, emocional y conductualmente a los retos que plantea la existencia.

Pero, por otra parte, entendemos que para quienes no dedican toda su actividad a la docencia (profesores de jornada parcial o "profesores hora"), es difícil asumir íntegramente las condiciones que requiere un cambio de paradigma tan grande, justamente porque se necesita un tiempo adicional para adquirir estas nuevas destrezas.

Adicionalmente, se perciben algunas incoherencias que dificultan el tránsito desde el paradigma de enseñanza tradicional, basado en la transmisión de conocimientos y experiencias, básicamente a través de la clase magistral, hacia uno más vivencial que debe entregar, adicionalmente, competencias. Algunas de estas incoherencias son las siguientes:

i) Las capacitaciones que suelen darse para aprender estos nuevos modelos o metodologías activas, colaborativas o participativas suelen enseñarse de la misma manera tradicional, o sea a través de discursos magistrales.

ii) Por otra parte, donde se han implementado estas nuevas metodologías de enseñanza activa, sin embargo, no se variado en las formas de evaluar, especialmente en las instancias más importantes de la carrera, conservándose los exámenes tradicionales, y más aún, manteniéndose el examen de grado en los mismos términos, es decir considerando, básicamente el viejo sistema memorístico/repetitivo.

Como decíamos, suele ocurrir que a los "profesores" universitarios, nadie nos ha enseñado fundamentos de pedagogía y menos aun metodologías activas, y ni siquiera hemos sido testigos de estos nuevos métodos, por lo que existe un amplio espacio para el

\footnotetext{
${ }^{10}$ Juan Carlos. Una triple alianza para un aprendizaje universitario de calidad. Madrid: Comillas, $2007 . \mathrm{P}$. 18.

${ }^{11}$ Ibíd. P. 19.
} 
desarrollo y la formación profesional de los actuales docentes, lo que pasa también por decisiones de política universitaria tendientes a profesionalizar la actividad, por lo menos en algún nivel elemental.

Por último, se debe tener presente que las experiencias activas o colaborativas deben entenderse, en un primer término, como técnicas excepcionales, pues es una experiencia nueva tanto para el estudiante como para el académico que se deber ir asumiendo progresivamente.

Lo más difícil: poner en práctica las metodologías colaborativas o participativas de enseñanza

Ya decíamos que a la mayoría de los académicos nadie nos ha enseñado a hacer clases o a impartir docencia, en términos amplios, y hemos intentado repetir lo que nosotros vivimos en las aulas, tratando en lo posible de corregir las falencias que reclamábamos de los maestros, con mayor o menor suerte. Y sobre las metodologías activas, apenas escuchamos hablar, pero no pudimos ser testigos de lo que ello significaba, ya que primaba el estricto sistema de la clase magistral, con académicos de primer nivel cuyos discursos era un privilegio escuchar, a los que muchas veces no se les podían interrumpir, por lo que la retroalimentación o participación de los estudiantes solía ser poco fluida, reduciéndose a un breve espacio de tiempo para preguntas, cuando queda tiempo para ello, y donde las posibilidades reales eran sólo para aquellos adelantados capaces de seguir con total atención el discurso o tenían algún entrenamiento previo que les permitía entablar algún diálogo mínimo con el expositor.

\section{Las experiencias activas o colaborativas como técnicas excepcionales}

Si bien, las metodologías colaborativas -que se basan en la idea de que el estudiante "colabore" o participe activamente en su propio aprendizaje-, han existido desde siempre, ello ha sido más bien excepcional en el ámbito de la enseñanza del Derecho duro y cuya implementación ha quedado entregada, básicamente, a las habilidades o carisma personal del docente y, mucho más excepcional aun, ha sido al diseño exprofeso de un curso completo en dicha especialidad. Sin embargo, esta realidad ha ido variando paulatinamente, desde que también el ejercicio de la profesión cambió, y debió incluir competencias adicionales adecuadas a los nuevos procedimientos de jurisdicción oral que se extienden por doquier.

Estimamos que un caso extremo de experiencia activa o colaborativa lo constituyen las Clínicas Jurídicas, que eventualmente pudiera tener una universidad, donde se interactúa con personas de verdad, que tienen problemas de verdad y donde el estudiante "hace lo que se puede" por salvar esta primera experiencia de su vida profesional debido a que, muchas veces, no se le han entregado anteriormente las herramientas necesarias para enfrentar adecuadamente esta experiencia demasiado real. A veces, esta situación se da sólo en la 
práctica profesional, siendo el sistema criticado por no brindar la atención adecuada a quienes no pueden pagar por la atención de un abogado con mayor "experiencia", dejando gran parte el peso del cumplimiento de la garantía constitucional del artículo 19 № 3, de la Constitución (defensa jurídica), en manos de estudiantes en práctica que nunca habían pisado un tribunal.

También el desempeño laboral de los estudiantes, realizado en forma paralela a los estudios, aunque no sea específicamente en el ámbito jurídico, se encarga de suplir las falencias de un sistema demasiado formal instaurado en la Universidad. Un escenario real, sin duda, proporciona un buen espacio para poner en práctica las teorías estudiadas y debatidas en el aula, en muchas áreas como la laboral o comercial. Lamentablemente esta posibilidad no suele ser común en nuestro medio ni en nuestra cultura, considerándose un bien que los estudiantes universitarios se dediquen exclusivamente a sus estudios.

\section{Guía práctica para intentar una experiencia colaborativa}

A continuación, y en base a la experiencia personal adquirida con el apoyo de algunos talleres de iniciación, quiero compartir una pauta o secuencia de pasos que ha sido fundamentales para mí y me han permitido avanzar en el nuevo paradigma. Es así, como he identificado, por lo menos, nueve elementos que me parecen indispensables de tener presente antes de iniciar una actividad participativa o colaborativa, cuyo objetivo es apoyar a los estudiantes en la adquisición de algunas de las competencias establecidas en el perfil de egreso de la carrera y donde el estudiante adquiere un rol fundamental en su propio aprendizaje.

\section{1) Perfil de egreso}

Es el compromiso que adquiere la institución de enseñanza para con sus egresados. Es en lo que debiera transformarse un estudiante luego de cursar el plan de estudio en que se ha matriculado. Este concepto es lo primero que debemos tener a la mano para intentar nuestro ejercicio, pues es a esos objetivos a los que debemos apuntar con nuestras enseñanzas. El problema es que a veces dicho perfil es tan extenso y amplio que se pierde de vista cual es la transformación fundamental que queremos lograr en el estudiante, o por el contrario es tan escueto o general que, prácticamente, no se distingue ningún elemento específico que le dé contenido al título genérico que se otorga. Ej: "Lo formamos para desempeñarse como abogado".

\section{2) Competencias}

Los mismos autores reconocen que "no hay una definición estandarizada de lo que es una competencia, pero puede considerársela como la integración de todos los saberes 
dirigida hacía una educación total del ser, basada en un aprendizaje significativo que le permita resolver los problemas que se le presenten a lo largo de la vida (Gonczi y Athanasou, 1996)" "12.

Esta definición demasiado amplia nos puede poner en problemas pues no podemos pretender entregar todo tipo de habilidades para enfrentar todos los problemas o situaciones que eventualmente pueden presentarse en la actividad profesional. De esta forma, es necesario hacer una selección adecuada de algunas competencias especialmente relevantes, de las cuales podríamos hacernos cargo en la cátedra particular que nos toca impartir.

Para ilustrar de mejor manera el concepto de competencia entregamos otras definiciones que nos pueden ayudar en este cometido:

- Intervención eficaz en diferentes ámbitos de la vida por medio de acciones en las que se movilizan, al mismo tiempo y de manera interrelacionada, conocimientos, procedimientos y actitudes (Antoni Zabala (2008). ${ }^{13}$

Un autor nos presenta una selección de definiciones de competencia, acotando que la dificultad en definir el concepto crece con la necesidad de usarlo, lo que pone de manifiesto que nos encontramos con un concepto que todavía se está construyendo. Estas definiciones se citan a continuación ${ }^{14}$ :

- Aplicación de las destrezas, conocimientos actitudes a las tareas o combinaciones de de las mismas según los niveles exigidos en condiciones operativas (Prescott, 1985)".

- "La competencia resulta de un saber actuar pero, para que se construya, es necesario un poder y un querer actuar (Le Boterf, 1994)"

- "Una construcción a partir de una combinación de recursos (conocimientos,saber hacer), cualidades o aptitudes, y recursos del ambiente (relaciones, documentos, informaciones y otras, que son movilizados para actuar (Le Boterf, 2000)".

- "La competencia es la capacidad de responder a exigencias realizando una tarea/actividad con resultados que incluye dimensiones cognitivas y no cognitivas (OCDE, 2003)".

La última definición que presentamos, que nos parece muy gráfica es:

capacidad de utilizar los conocimientos y habilidades, de manera transversal e interactiva, en contextos y situaciones que requieren la intervención de conocimientos vinculados a diferentes saberes, cosa que implica la comprensión, la reflexión y el discernimiento teniendo en cuenta la dimensión social de cada situación ${ }^{15}$.

\footnotetext{
12 OBAYA VALDIVIA, Adolfo y PONCE PÉREZ, Ruben G. "Evaluación del aprendizaje basado en el desarrollo de competencias". En: Contactos. 2010, núm. 76. Pp. 31-37. Disponible en web: http://www.izt.uam.mx/contactos/n76ne/competencias.pdf [consultado en abril 2012].

${ }^{13}$ LORENTE CATALÁN, Eloisa y LACASA CLAVER, Enrique. "Desaprender para avanzar. Revisar para cambiar”. En: BLÁZQUEZ, Domingo y SEBASTIANI, Enric (editores). Enseñar por competencias en educación física. Barcelona: INDE, 2009. P. 24.

${ }^{14}$ SEBASTIANI, Enric, BLÁZQUEZ, Domingo y BORRACHINA, Julio. "Concepto y naturaleza de las competencias". BLÁZQUEZ, Domingo y SEBASTIANI, Enric. Ibíd. P. 42.

${ }^{15}$ BLÁZQUEZ, Domingo y SEBASTIANI, Enric (editores). Ibíd. P. 212.
} 
La determinación de las competencias que se entregarán a un estudiante que cursa una determinada carrera es un dato que viene dado para el académico, pues es un elemento que corresponde al diseño de la carrera.

Al igual que lo que ocurre con el perfil, a veces, estas competencias no suelen estar adecuadamente especificadas en nuestros programas de clase, sea porque son muchas o, por el contrario, son demasiado genéricas. Seleccionamos algunos ejemplos que nos parecen transversales a cualquier carrera de Derecho y que podríamos tener en consideración cuando elaboremos nuestro ejercicio práctico destinado, precisamente, a entrenar aquella competencia elegida:

- Transmitir conocimientos jurídicos mediante publicaciones y exposiciones orales dirigidas a especialistas o al público en general;

- Comprender y analizar críticamente textos y documentos vinculados a la profesión y a las disciplinas asociadas a ellas;

- Expresarse, oralmente y por escrito, de manera eficaz, utilizando un lenguaje correcto y el manejo de un amplio repertorio de conceptos jurídicos;

- Localizar normas e instituciones de ordenamientos jurídicos extranjeros, supranacionales e internacionales;

Estas competencias seleccionadas dicen relación con habilidades para la investigación, el análisis de textos, la expresión oral y escrita. Luego habrá que seleccionar qué tipo de ejercicio permite entrenar de mejor forma alguna de ellas.

\section{3) Programa de estudio}

Es la herramienta de trabajo del profesor y debiera ser también la del alumno, es la guía o pauta que nos acompaña en todo el proceso formativo en una determinada área en que se ha parcelado el conocimiento.

Hay que tener presente que un programa tradicional no puede ser el mismo para un sistema educativo basado en competencias, pues básicamente se trata de enseñar a los estudiantes a que aprendan por sí mismos, especialmente, a proveerse ellos mismos del material que se requiere para el ejercicio de la profesión. De esta forma, ya no se necesitan programas enormes que pretendan llenar de información y datos a los estudiantes, para que los retengan en la memoria (cuestión que, en todo caso, nunca está demás para quienes tienen capacidad para ello), sino a solucionar los problemas a los que suele enfrentarse un abogado en la vida real, entre ellos a proveerse de buena información, focalizada al problema que se debe atender en el momento. Se trata de saber dónde buscar la información, cómo usarla, cómo interpretarla, cómo argumentar y en definitiva cómo resolver problemas en forma eficiente, al menor costo y en forma ética. 


\section{4) Materia específica sobre la que se aplicará el método activo}

Continuando con nuestra guía práctica para novatos, el siguiente paso es seleccionar una materia en particular que será la que nos servirá para la aplicación de la metodología activa. Ya en etapas posteriores se podrá ir incorporando en forma paulatina el resto del curso o una parte de él.

La materia elegida permite incorporar conocimientos o derecho duro, pero también competencias, de aquellas que ya habíamos señalado, o sea habilidades para la investigación, la redacción de documentos, el análisis de normas, etc.

\section{5) Selección de un ejercicio específico}

Los ejercicios posibles son bastos, a modo de ejemplo citamos los más conocidos: estudio de casos (con distintos grados de complejidad), debates amplios, discusión dirigida, juego de rol, lluvia de ideas, mapa conceptual, mesa redonda, proyecto de trabajo, simulación, solución de problemas, investigación, acopio y análisis de jurisprudencia, etc. Estos y muchos otros métodos de enseñanza activa permiten al estudiante participar directamente en la construcción de su propio conocimiento y formación y en el entrenamiento de competencias.

Estos ejercicios, según decíamos, pueden ser muy simples o muy complejos dependiendo de la cantidad de elementos que se incorporen. La configuración de cada uno de los ejercicios señalados y de otros se puede encontrar fácilmente en la web o se pueden ir creando otros en la medida que se adquiera dicha "competencia" con la práctica y la experiencia.

Algunos de estos métodos han pasado a ser mucho más que una nueva forma de enseñar, y se consideran una fórmula que debería pernear e inspirar toda la enseñanza universitaria. Así, se estima que:

el aprendizaje basado el problemas (ABP) se perfila como uno de los enfoques más innovadores en la formación profesional y académica actual, conquistando cada vez más espacio en las principales universidades del mundo. Tras este movimiento se encuentra la búsqueda de nuevos modelos de producción y organización del conocimiento, de acuerdo con las demandas y necesidades de las sociedades contemporáneas, así como la denominada sociedad del conocimiento. El sistema universitario no está exento de los cambios sociopolítico-económicos de las últimas décadas y, por lo tanto, necesitan reinventarse para mantener el destacado lugar que ocupa en la sociedad desde hace trescientos años. Paradójicamente, esta reinvención depende tanto de la capacidad de conservar sus características de excelencia y de producción de conocimientos, como de la adaptación a las nuevas exigencias de la sociedad, la cultura y la ciencia ${ }^{16}$.

\footnotetext{
${ }^{16}$ ARAÚJO, Ulises y SASTRE, Genoveva (coordinadores). El aprendizaje basado en problemas. Una nueva perspectiva de la enseñanza en la universidad. Barcelona: GEDISA, 2008. P. 9.
} 
Las perspectivas del ABP colocan al/la alumno/a en el núcleo del proceso educativo, otorgándole autonomía y responsabilidad por el aprendizaje propio a través de la identificación y análisis de los problemas y de la capacidad para formular interrogantes y buscar informaciones para ampliarlos y responderlos; y a partir de ahí, para recomenzar el ciclo con nuevas cuestiones, procesos de aprendizaje y cuestionamiento de la realidad. Detrás de estos procesos educativos hay un cambio de enfoque en la enseñanza superior, que deja de centrarse en la enseñanza para priorizar los procesos de aprendizaje ${ }^{17,}$.

Otra faceta esencial del ABP consiste en tomar problemáticas concretas y situaciones Reales como puntos de partida para los procesos de aprendizaje, que además de resultar estimulante, contribuye al desarrollo de la responsabilidad social y aporta una formación sólida para el ejercicio profesional. De esta manera, consideramos que la adopción del $\mathrm{ABP}$ en las instituciones educativas es una poderosa herramienta para la formación de profesionales y científicos, en sociedades que exigen la estructuración de conocimientos sólidos y profundos de la realidad y la construcción de la justicia social ${ }^{18}$.

\section{6) Selección de la oportunidad}

Una vez diseñado nuestro ejercicio inicial debemos pensar en qué momento se aplicará. Puede ser al comienzo de la clase, durante o al finalizar la clase (ejemplo, un debate), ello dependerá de la complejidad del ejercicio diseñado o seleccionado. También se puede dedicar una sesión completa en la semana a este tipo de ejercicios (analizar un caso) o varias sesiones, o un ejercicio para finalizar el semestre. En esta idea de entregar pautas para novatos, estimo que hay que centrarse en un ejercicio muy simple para comenzar, el que con la experiencia adquirida se puede ir complejizando, en la medida que todos van adquiriendo más práctica en ello. Por ejemplo, un debate simple y libre sobre un tema en particular, el que luego se puede hacer más complejo si se asignan posiciones a los estudiantes que deben defender en el debate, luego se pueden asignar roles concreto (juez, defensor, fiscal, víctima, etc.), lo que puede terminar con una simulación compleja de un juicio.

\section{7) Ejemplo de un ejercicio específico}

Presentamos a continuación un ejercicio muy sencillo, donde se pretende entrenar competencias vinculadas con el análisis de normas, expresión oral y escrita y trabajo en equipo, cuyo fin es ejercitar la tolerancia y las habilidades sociales de los estudiantes, de manera que puedan compartir con cualquier compañero, aunque no sean aquellos de su círculo más cercano.

Se trata de enfrentar un caso típico de Derecho Internacional Privado, donde no hay una solución única, pues lo importante no es el resultado, sino el proceso. El ejercicio se desarrolla en una clase bajo la supervisión del profesor y un ayudante, en el supuesto que el curso no pasa de los 20 alumnos.

\footnotetext{
${ }^{17}$ ARAÚJO, Ulises y SASTRE, Genoveva. Ibíd. P. 11.

${ }^{18}$ Ibíd.
} 
El ejercicio concreto es el siguiente: en grupos de tres estudiantes (formados por el profesor) se debe resolver una situación vinculada a la teoría del reenvío, matrimonio, sucesión y excepciones a la aplicación del derecho extranjero. Los resultados se exponen al curso y se entrega informe escrito preliminar. La evaluación del trabajo en grupo será conforme a la rúbrica adjunta.

Caso: un yemení muere en España en un viaje destinado a la supervisión de los negocios que allí poseía, donde tenía tres esposas, dos hijos, y una concubina, dejando también negocios y bienes en Chile, donde además deja un hijo chileno quien demanda la distribución de dichos bienes, habiendo un testamento que favorece a otras personas en forma mínima.

Pregunta: siendo usted. el juez ¿por qué ley se regla la sucesión y por qué? ¿Qué instituciones son problemáticas en el caso y por qué?

\section{8) Rúbrica}

Esta, en mi opinión, es una de las partes más importantes en la enseñanza basada en competencias, pues permite orientar al estudiante en cuanto a la forma en que será evaluado en su participación en el ejercicio práctico justamente para entrenar una o varias competencias. La rúbrica o matriz de evaluación es una tarea que corresponde al profesor, para lo cual también existe un variado abanico de posibilidades y modelos disponibles en la web. Se trata de una herramienta fundamental e ineludible para la evaluación, que tiene por objeto comunicar a los estudiantes qué desempeños se medirán y los estándares de desempeño esperado, de manera que ellos sepan a qué atenerse y puedan evaluar sus propios avances en la medida que elaboran sus tareas. Las fórmulas son múltiples tanto para medir el nivel de desempeño o de cumplimiento, como los desempeños mismos que exigen y que se pretenden medir.

Presentamos un ejemplo de matriz de evaluación para nuestro ejercicio de trabajo en grupo o equipo, donde se indican los desempeños que permitirían entrenar competencias vinculadas al análisis de normas, expresión oral y escrita, tolerancia y habilidades sociales de los estudiantes. 


\begin{tabular}{|c|c|c|c|c|}
\hline $\begin{array}{l}\begin{array}{r}\text { Nivelde } \\
\text { desempeño }\end{array} \\
\text { Desempeños } \\
\text { a medir }\end{array}$ & Excelente 4 & bueno 3 & Insuficiente 2 & Necesita mejorar 1 \\
\hline $\begin{array}{l}\text { Participación } \\
\text { equitativa de todos } \\
\text { los integrantes }\end{array}$ & $\begin{array}{l}\text { Participa } \\
\text { ponderadamente } \\
\text { escvchando a los } \\
\text { demas e } \\
\text { incentivando para } \\
\text { que todos los } \\
\text { miembros del } \\
\text { grupo puedan } \\
\text { expresar sus } \\
\text { opiniones en forma } \\
\text { libre y espontánea }\end{array}$ & $\begin{array}{l}\text { Se expresa con } \\
\text { fluidez y permite } \\
\text { que todos puedan } \\
\text { expresarse. }\end{array}$ & $\begin{array}{l}\text { Tiende a centrar el debate } \\
\text { en sus propios } \\
\text { argumentos, sin } \\
\text { considerar las opiniones } \\
\text { del resto. No incentiva } \\
\text { para que todos los } \\
\text { miembros del grupo } \\
\text { puedan opinar en forma } \\
\text { libre y espontanea. O sus } \\
\text { intervenciones son } \\
\text { esporadicas y fuera da } \\
\text { contexto. }\end{array}$ & $\begin{array}{l}\text { No permite el debate y } \\
\text { la libre expresión de los } \\
\text { demás miembros del } \\
\text { grupo, pues sólo admite } \\
\text { sus propios argumentos. } \\
\text { O no participa del } \\
\text { debate siendo un mero } \\
\text { espectador }\end{array}$ \\
\hline $\begin{array}{l}\text { Respeto y } \\
\text { tolerancia a la } \\
\text { opinión de los } \\
\text { demas }\end{array}$ & $\begin{array}{l}\text { Acepta todos los } \\
\text { puntos de vista y } \\
\text { los pondera } \\
\text { objetivamente con } \\
\text { los antecedentes de } \\
\text { que se dispone. }\end{array}$ & $\begin{array}{l}\text { Escucha los } \\
\text { planteamientos de } \\
\text { los demas pero } \\
\text { hace ver que no } \\
\text { son los que } \\
\text { tradicionalmente } \\
\text { aceptados en la } \\
\text { comvidad de que } \\
\text { se trata por lo } \\
\text { herrado de sus } \\
\text { bases. }\end{array}$ & $\begin{array}{l}\text { No acepta todos los } \\
\text { planteamientos } \\
\text { descalificando aquellas } \\
\text { opiniones que miestran } \\
\text { diferencias o son opvestas } \\
\text { a las svyas. }\end{array}$ & $\begin{array}{l}\text { Descalifica a las } \\
\text { personas por emitir } \\
\text { opiniones que no } \\
\text { concuerdan con las } \\
\text { suya. }\end{array}$ \\
\hline $\begin{array}{l}\text { Manajo de } \\
\text { vocabulario } \\
\text { adecuado y de } \\
\text { conceptos } \\
\text { juridicos } \\
\text { fundamentales } \\
\text { Expresión claray } \\
\text { concreta de ideas }\end{array}$ & $\ldots \ldots \ldots \ldots \ldots \ldots \ldots$ & f...................... & $\ldots \ldots \ldots \ldots \ldots \ldots \ldots$ & $\ldots \ldots \ldots \ldots \ldots \ldots \ldots$ \\
\hline $\begin{array}{l}\text { Capacidad para } \\
\text { negociar }\end{array}$ & .......................... & $\ldots \ldots \ldots \ldots \ldots \ldots \cdots \cdots$ & ......................... & $\ldots \ldots \ldots+\cdots \cdots \cdots \cdots$ \\
\hline
\end{tabular}

El puntaje máximo para los cuatro desempeños son dieciséis puntos, que correspondería a un siete, y el mínimo son cuatro, que puede ser el equivalente a un dos o a un uno. Estas escalas son normales como cualquiera que se use en una prueba escrita. La matriz se puede ampliar para abarcar otros desempeños o más estándares de desempeños y también reducir, la idea es que el docente la adapte al trabajo que piense desarrollar. 


\section{9) Rol del profesor en el ejercicio}

El profesor guía, comparte, orienta y por supuesto observa y evalúa conforme a la rúbrica adjunta. Para ello debe contar con los ayudantes necesarios que le permitan manejar los grupos formados de acuerdo al mayor tamaño. También es bueno considerar la infraestructura, que aunque mínima permita formar los grupos, con espacio suficiente para poder interactuar en forma cómoda e independiente.

Todo el ejercicio puede irse complejizando y terminar en una simulación completa de un juicio, con roles definidos, presentado públicamente en la sala del juicio oral (si la hay).

Sobre el nuevo rol del profesor universitario en los tiempos que corren habrá que discutirse bastante para ver cómo se puede conjugar adecuadamente teoría y realidad, Por ello la innovación en esta área es una tarea que ya partió en el viejo continente pero que nosotros aun no acabamos de entender ${ }^{19}$.

\section{Conclusión}

Como he señalado antes, estas líneas pretenden mostrar una experiencia que puede servir de guía práctica "de emergencia" para quienes se inician en las metodologías activas, más bien basada en la experiencia personal y la formación recibida en distintas instancias de capacitación entre ellas el "Certificado en educación superior", impartido por Laureate International Universities (on line), lo que me ha permitido ir construyendo mis propias destrezas iniciales en estas metodologías, especialmente la de perder el miedo al cambio, de ahí, en adelante, no hay límites, y cada profesor con su experiencia puede ir construyendo sus propios ejercicios, ser creativo y salir del típico "control de lectura" como la actividad más "activa" del curso. Por cierto que estás líneas no pretenden reemplazar la labor de los expertos y la adecuada y permanente capacitación que cada docente debe recibir, con la idea de profesionalizar la enseña universitaria, al menos en un porcentaje aceptable, sobre todo en nuestra área, donde la mayoría somos aficionados en materia de pedagogía.

Mi conclusión final es que se deben ir alineando los elementos fundamentales del currículo: perfil, competencias, metodología, ejercicios y evaluación, esa, estimo, es la clave para iniciar el camino.

\footnotetext{
${ }^{19}$ Sobre este tema se puede consular: MARGA LEF, Leonor y TORNÉ, Emilio (editores). Estrategias de innovación docente para favorecer el aprendizaje autónomo de los estudiantes de la Universidad de Alcalá. Alcalá de Henares: Universidad de Alcalá, 2007; y CASANOVA, María Antonia. Diseño curricular e innovación educativa. $2^{\mathrm{a}}$ ed. Madrid: La muralla, 2009.
} 


\section{Referencias bibliográficas}

ARAÚJO, Ulises y SASTRE, Genoveva (coords). El aprendizaje basado en problemas. Una nueva perspectiva de la enseñanza en la universidad. Barcelona: GEDISA, 2008. P. 205.

BUJAN, Federico. La reforma de los estudios de derecho. El nuevo plan de estudio: su valoración y análisis histórico y comparado. Madrid: Dykinson, 1992. P. 250.

CASANOVA, María Antonia. Diseño curricular e innovación educativa. $2^{\mathrm{a}}$ ed. Madrid: La muralla, 2009. P. 260.

FIGUEROA YÁÑEZ, Gonzalo. "El estado actual de la docencia y de la investigación en derecho". Documento de trabajo Nº 283, Santiago: Corporación de Promoción Universitaria, 1983. P. 48.

LIMODIO, Gabriel. Los principios y la enseñanza del derecho privado. Buenos Aires: Editorial de la Universidad Católica Argentina, 2008. P. 454.

LORENTE CATALÁN, Eloísa y LACASA CLAVER, "Desaprender para avanzar. Revisar para cambiar", en Blázquez, Domingo y Sebastián, Enric (editores). Enseñar por competencias en educación física. Barcelona: INDE, 2009. P. 20.

MARGA LEF, Leonor y TORNÉ, Emilio (editores). Estrategias de innovación docente para favorecer el aprendizaje autónomo de los estudiantes de la Universidad de Alcalá. Alcalá de Henares: Universidad de Alcalá, 2007. P. 254.

REYES, María y LEAL, María. El aprendizaje del derecho en el nuevo espacio europeo de enseñanza superior. Sevilla: Mergablum, 2006. P. 181.

SEBASTIANI, Enric, BLÁZQUEZ, Domingo y BORRACHINA, Julio. "Concepto y naturaleza de las competencias". En: BLÁZQUEZ, Domingo y SEBASTIANI, Enric (editores). Enseñar por competencias en educación física. Barcelona: INDE, 2009. P. 20.

STAINBACK, Susan y STAINBACK, William. Aulas inclusivas. Un nuevo modo de enfocar y vivir el currículo. $4^{\mathrm{a}}$ ed. Madrid: NARCEA, 2007. P. 295.

TORRE PUENTE, Juan Carlos. Una triple alianza para un aprendizaje universitario de calidad. Madrid: Comillas, 2007. P. 263. 